\title{
ESTEBAN MOLINA
}

\section{EL CUERPO Y LA IDEA DE SUJETO ENCARNADO EN LA FENOMENOLOGÍA DE LA PERCEPCIÓN DE MERLEAU- PONTY}

THE BODY AND THE IDEA OF THE EMBODIED SUBJECT ON MERLEAU-PONTY'S PHENOMENOLOGY OF PERCEPTION

estcarmolina@gmail.com

Recepción: 06/04/2021

Aceptación: 11/06/2021

\section{RESUMEN}

Dada la vigencia y la importancia del pensamiento de M. Merleau-Ponty en torno a la cuestión de la corporalidad, el trabajo se focaliza sobre la Fenomenología de la percepción (1945) y expone, como resultado del vínculo 'cuerpo' y 'mundo', a la disposición existencial como aquella que establece la significación de los actos psicológicos. En esta línea, el trabajo apunta a seguir pensando la noción de 'sujeto encarnado' en la filosofía de Merleau-Ponty y, en conexión a ello, la manera en la cual habitamos corporalmente en el mundo a partir de la conceptualización de cuerpo como vehículo del ser en el mundo.

\section{PALABRAS CLAVES}

M. Merleau-Ponty, percepción, sujeto encarnado, cuerpo, fenomenología.

\section{ABSTRACT}

Given the current validity and importance of Merleau-Ponty's reflection on the matter of corporality, his contribution is focused on Phenomenology of perception and exposes because of the bind between 'body' and 'world' to the existential disposition as the one that establishes the significance of psychological acts. The author aims to keep thinking the notion of the embodied subject and the way in which we bodily inhabit the world thereafter the conceptualization the body as a vehicle of the being in the world.

\section{KEYWORDS}

M. Merleau-Ponty, perception, embodied subject, body, phenomenology. 


\section{INTRODUCCIÓN}

Nuestro objetivo general consiste en situarnos en el análisis de la Fenomenología de la percepción (Phénoménologie de la Perception, 1945), para comprender la perspectiva de Maurice Merleau-Ponty acerca de la dimensión corporalidad-espacialidad. Así, pues, desde la óptica de la fenomenología descriptiva, la finalidad es desarrollar la idea de cuerpo merleaupontyana, en el mencionado periodo, y ver la conexión que establece con el mundo y las cosas por medio de la percepción. Por consiguiente, este análisis nos permitirá explicitar el modo en que habitamos corporalmente el mundo. Nuestro trabajo consta de las siguientes partes: en primer lugar, realizaremos una descripción introductoria sobre la cuestión a tratar, la cual cuenta ya con un precedente en la obra La estructura del comportamiento (La structure du comportement, 1942); en segundo lugar, nos detendremos a desarrollar la idea de 'sujeto encarnado' y el concepto 'percepción' en Merleau-Ponty; dando paso a un tercer lugar, que tematiza de la noción 'cuerpo' en la Fenomenología de la percepción, cuyo análisis finaliza con un punto de vista aportado por Bernhard Waldenfels acerca de habitar corpóreamente el mundo, que a nuestro criterio enriquece lo explicitado por Merleau-Ponty.

A continuación, comenzaremos el presente trabajo con una breve referencia a $L a$ estructura del comportamiento (La structure du comportement, 1942), la cual nos dará acceso a abordar y vincular la cuestión de la percepción con la problemática alma-cuerpo. Este breve paso nos brindará la posibilidad de visualizar el enfoque de Merleau-Ponty en relación al ser humano y el modo en que lo vincula con la capacidad de percibir, a partir de la perspectiva del espectador externo.

\section{CUERPO, MUNDO, SERES Y COSAS}

En La estructura del comportamiento, Merleau-Ponty indaga particularmente en torno a la idea de naturaleza y conciencia, sin embargo, aparecen otras nociones a tener en consideración. Tal es el caso con la noción de 'estructura' o 'forma' -noción que el fenomenólogo francés utiliza de manera indiferenciada. Merleau-Ponty expresa que "[...] el comportamiento no es una cosa, pero tampoco es una idea; no es la envoltura de una pura conciencia y, como testigo de un comportamiento, no soy una pura conciencia. Es lo que queríamos expresar con forma" (Merleau-Ponty, 1953, p. 183). Esa conexión se puede cotejar con el tratamiento que despliega Merleau-Ponty sobre las descripciones de distintas formas del comportamiento, las cuales aparecen descritas de manera gradual o de forma escalonada. En otras palabras, Merleau-Ponty describe las formas, en un primer momento, sincréticas, las cuales, en un segundo momento, las describe bajo la forma de amovible, para luego describirlas un tercer momento, más abarcativo, como formas simbólicas, siendo esta última aquella que exige la actividad humana. ${ }^{1}$ Esta descripción muestra, por un lado, el desarrollo de integración de una forma simple en una más compleja y, como espejo, la aparición de una forma superior a partir de otra inferior; por otro, la manera como Merleau-

${ }^{1}$ Capítulo II, "Los comportamientos superiores", en el especial en el punto "Las estructuras del comportamiento" (1953, pp. 140-183). 
Ponty va proyectando la idea de encarnación a partir de ese paso a otro entre las diferentes formas. Los animales pueden encontrarse o situarse en cualquiera de las tres formas, siguiendo la explicación de Merleau-Ponty, pero la que exige el hombre es la forma simbólica. Con respecto a esto, en las descripciones de las distintas formas de comportamientos podemos observar y trazar un análisis cómo la totalidad perceptiva estriba y conecta con la significación funcional. Por ello, para nuestros fines, es importante resaltar lo descripto, ya que la noción de estructura o forma está conectada con otra idea importante, a saber, la idea de 'totalidad'. ${ }^{2}$ En efecto, esta idea de totalidad concierne a la idea del ser vivo y a sus respectivos comportamientos.

En el último capítulo, "Las relaciones entre el alma y el cuerpo, y el problema de la conciencia perceptiva", Merleau-Ponty indaga en torno a la unidad del hombre. Allí expresa la idea que al cuerpo humano no le han quitado aún sus atributos. En otras palabras, significa que la unidad del ser humano aún no ha sufrido ruptura que trae como consecuencia la transformación del hombre en una máquina. De hecho, el cuerpo mismo no es captado como un dispositivo externo, sino como el envoltorio viviente de nuestras intenciones. "Nuestras intenciones -sostiene Merleau-Ponty- encuentran en los movimientos su vestimenta natural o su encarnación y se expresan en ellos como la cosa se expresa en sus aspectos perspectivos" (Merleau-Ponty, 1953, p. 263). En referencia a esto es que Merleau-Ponty concibe aquí la idea de sujeto como aquel que vive en un mundo de experiencia, mediado por su cuerpo e interactúa con los seres y las cosas en un mismo ámbito. ${ }^{3}$ Merleau-Ponty amplia esta idea de la siguiente manera:

El sujeto no vive en un mundo de estados de conciencia o de representaciones, desde donde creería poder, por una especie de milagro, actuar sobre las cosas exteriores o conocerlas. Vive en un universo de experiencia; en un medio neutro respecto a las distinciones substanciales entre el organismo, el pensamiento y la extensión, en un comercio directo con los seres, las cosas y su propio cuerpo. El ego, como centro del que irradian sus intenciones, el cuerpo que las lleva, los seres y las cosas a las que ellas se dirigen no están confundidos; pero no son más que tres sectores de un campo único. Las cosas son cosas, es decir, trascendentes respecto a todo lo que sé de ellas, accesibles a otros sujetos percipientes, pero justamente consideradas como tales, y como tales, momento indispensable de la dialéctica vivida que las abraza. (Merleau-Ponty, 1953, pp. 263-264)

Merleau-Ponty explicita, así, la idea de cuerpo como transporte de nuestras intenciones. No obstante, el cuerpo generalmente es un cúmulo de senderos ya proyectados, es el soporte dialéctico logrado sobre el cual se realiza una puesta en forma extraordinaria. El cuerpo, por lo tanto, es una significación y únicamente tiene sentido para la mirada de

\footnotetext{
${ }^{2}$ En este sentido, Ralón agrega: “[a]sí como la noción de integración es pensada en vista a la dialéctica humana, la noción de totalidad aparece como un mixto en el que la forma o la estructura compone lo proveniente de la idea y lo proveniente de la cosa. Más precisamente, la forma que da unidad a los diferentes tipos de comportamiento aparece como una idea encarnada en una porción de extensión" (2015, p. 166).

${ }^{3}$ En Filosofía y lenguaje, diversificará la cuestión ampliando la noción de cuerpo como un sistema de símbolos. En este sentido, dice: “[e]1 cuerpo es el portador de un número indefinido de sistemas simbólicos cuyo desarrollo intrínseco excede, seguramente, la significación de los gestos 'naturales', pero que se destruyen si el cuerpo deja de puntualizar su ejercicio y de instalarlos en el mundo y en nuestra vida" (1969, p. 16).
} 
una conciencia. En efecto, el cuerpo es el aspecto perceptivo de todas las conciencias que conocemos por medio del cuerpo. Pero, ¿qué entiende Merleau-Ponty acerca de lo percibido? Para él, lo percibido es su significación y no un resultado de la operatividad cerebral. Sin embargo, podría decirse que toda dialéctica particular tiene a modo de expresión fases cerebrales, las cuales ella desconoce. Pese a ello, ¿qué entiende aquí por significación? ¿Es la significación lo que media y liga la actualidad y la virtualidad entre mí y un observador situado? ¿Cómo se conforma una estructura de experiencia? Veamos la explicación que da:

[...] cada vez que se actualizan en mi campo de conciencia tales fenómenos sensibles, un observador situado convenientemente vería en mi cerebro tales otros fenómenos que no se pueden dar a mí mismo en el modo de la actualidad. [...] Puedo, a partir del espectáculo actual que se me da, representarme en el modo de lo virtual, [...] ciertos fenómenos retinianos y cerebrales que localizo en una imagen virtual de mi cuerpo. El hecho de que el espectador y yo mismo estemos ligados uno y otro a nuestro cuerpo equivale, en suma, a esto: que lo que puede dárseme en el mundo de la actualidad, como una perspectiva concreta, sólo se le da a él en el modo de la virtualidad, como una significación, e inversamente. Mi ser psicofísico total [...] es, en suma, un entrelazamiento de significaciones tal que, cuando algunas de ellas se perciben y pasan a la actualidad, las otras se alcanzan sólo virtualmente. Pero esta estructura de experiencia es semejante a la de los objetos exteriores. Más aún, se presuponen mutuamente. Si para mí hay cosas, es decir, entes perspectivos, en su aspecto perspectivo mismo está incluida la referencia a un punto desde el cual los veo. Pero estar situado en un cierto punto de vista es necesariamente no verlo a él mismo, poseerlo como objeto visual sólo en una significación virtual. La existencia de una percepción exterior, la de mí cuerpo, y 'en' ese cuerpo, la de fenómenos para mí imperceptibles, son, pues, rigurosamente sinónimos. Entre una y otra no hay relación de causalidad. Son fenómenos concordantes. (Merleau-Ponty, 1953, pp. 299-300)

A nuestro juicio es importante destacar la relación que establece Merleau-Ponty respecto a considerar la concordancia de la experiencia de una percepción exterior con la de mí cuerpo, puntualizar sobre la concordancia de esos fenómenos aporta para comprender la noción de percepción que traza hasta la publicación de la Fenomenología de la percepción. Ciertamente, Merleau-Ponty expresa que percibimos las cosas de manera directa, sin que mi cuerpo oficie de membrana entre las cosas y yo. Nuestros ojos no son un cúmulo de tejidos y de órganos turbios y diáfanos sino los medios de nuestra mirada. Vemos con nuestros ojos. Vemos mediante nuestros ojos.

Supuesto esto, observamos cómo la indagación filosófica de Merleau-Ponty acerca de la noción 'cuerpo' ahonda en la concepción objetivista del cuerpo, y en la traslación de esta concepción a una configuración del comportamiento, cuyo primer momento la desvincula de la estructura de las cosas y pasa, en un segundo momento, a restituirla como totalidad, para comprenderla en la percepción del espectador. En verdad, lo que trata de establecer Merleau-Ponty en La estructura del comportamiento es la funcionalidad del cuerpo y el vínculo con el exterior. Para ello, determina lo siguiente: al expresar que nuestra actividad refleja, involuntaria o cotidiana, posee un interior, ya que atestigua la conexión entre los estímulos y su significación en una coyuntura -incluso adaptándose a pequeños 
cambios de esta coyuntura-, se debe a que la misma actividad no tiene el sentido de la misma manera que lo hace la inteligencia, cuyo resultado expresa que el sujeto del reflejo, de la involuntariedad y de la cotidianidad, no es un espíritu. En un texto publicado con posterioridad, bajo el nombre de Parcours Deux 1951-1961, el cual reúne una serie de publicaciones entre las cuales se destaca "Titres et travaux. Projet d'Enseignement", Merleau-Ponty habla sobre la significación ambigua que tiene el cuerpo que funciona. Es decir, la ambigüedad radica en que no se lo puede considerar como una idea ni tampoco como una sumatoria de partes. De hecho, ningún autor serio, expresa Merleau-Ponty, cuestiona su existencia si el significado de las localizaciones cerebrales se ha transformado. En otras palabras, partiendo del supuesto que las consideraciones de cualidad o de valor propician la comprensibilidad del funcionamiento del organismo, éste no obstante se extiende en el espacio, adaptándose a las condiciones locales e instalándose en el cuerpo, y, de hecho, permanece sensible a toda alteración somática (Merleau-Ponty, 2000, pp. 9-35). Es así como Merleau-Ponty admite que no puede abordarse un examen del cuerpo humano como si fuera una cosa, es decir, el análisis del cuerpo no debe quedar aislado en un mero examen acerca de sí mismo. Es más, señala que este examen nos sitúa ante la comparecencia de una conexión con el ambiente. De manera que el modo de vincularse con el ambiente no es atribuible a un espíritu, sino a una conciencia capaz de percibir con una lógica propia. Sin embargo, aún el sujeto no ha podido adueñarse de la significación ideal ya que se encuentra implicado en las configuraciones específicas. Así, pues, el comportamiento trasluce una heterogeneidad de estructuras de modo valorativas diferentes, cuyo resultado evidencia cada vez más el predominio de las condiciones originadas desde el interior, conforme a no ponerse sobre los distintos órdenes animales.

No obstante, nuestra perspectiva no nos permite visualizar todavía a 'alguien', ya que continuamos posicionados desde la perspectiva del 'espectador extraño'; pues, solamente podremos 'verlos' a partir de que el espectador extraño sea impelido por el lenguaje a mantener con quien habla vínculos de correspondencia verdadera, además de reconocerse de modo legítimo con aquello que percibe. Por consiguiente, presentado resumidamente esta concepción merleaupontyana, obtenemos el respaldo para continuar con nuestra explicitación con un análisis de la percepción en el individuo, capaz esta de aclarar una concepción subjetiva del cuerpo. Para ello pasaremos a la Fenomenología de la percepción. A continuación nos detendremos a explicitar dos importantes cuestiones implícitas en nuestro trabajo, que dado a su importancia corresponde profundizarlos en un trabajo aparte, estas son: primeramente, el lugar de la 'percepción' en el pensamiento de Merleau-Ponty, y, por último, la idea fenomenológica de 'sujeto encarnado', ambas cuestiones puntualmente tratadas en la Fenomenología de la percepción. Veamos de qué tratan.

\section{PERCEPCIÓN, CUERPO Y MUNDO}

Merleau-Ponty considera que un análisis pormenorizado del cuerpo nos llevaría a ordenar algunas cuestiones filosóficas, particularmente cuestiones propias de los ámbitos 
ontológicos y fenomenológicos. Así, en el ideario filosófico merleaupontyano se sitúa la percepción ${ }^{4}$ como eje fundamental constitutivo entre la realidad y nuestro interior. De esto deriva lo siguiente: "[1] a percepción no es una ciencia del mundo, no es ni siquiera un acto, una toma de posición deliberada, sino que es el fondo sobre el que todos los actos se destacan y está presupuesta por ellos" (Merleau-Ponty, 1957, p. IX). Esto quiere decir que la idea de percepción se procura un significado epistemológico, alrededor de la cuestión de la verdad del objetivismo y del subjetivismo, impregnada por las participaciones del cuerpo con que se encuentran limitadas. De este modo, con la percepción se unen sujeto y objeto en un tipo de experiencia que encarna una completa relación con el mundo, el cuerpo mediante. Por otro lado la manera de acceder justamente al acontecimiento de esta variación, a través del acto de la percepción, es por el hecho de haberlo encarnado particularmente. En ese marco, González y Jiménez expresan: “[p]or la percepción se revela el ser, se descubre frente a nosotros y nos dice que, en virtud del ejercicio de la percepción, el hombre no puede desligarse nunca del ser" (González y Jiménez, 2011, p. 116). Por tal motivo, para ambos autores, podemos decir que una revelación es sinónimo de una percepción, es decir, es una "revelación del ser". 5

En verdad, Merleau-Ponty expresa que sus análisis lo conducían a un imaginario del cuerpo, aunque implícitamente formaba parte de un imaginario originario del mismo azar de la existencia. Así, la idea de Gestalt nos llevaba hacia un retroceso, por su propio peso conceptual de matiz hegeliano. Se decía que aquello exterior de un concepto era la naturaleza. No obstante, el concepto como tal no posee exterior, precisamente, por tal motivo, existía una necesidad de reflexionar la Gestalt como una unión de la naturaleza y de la idea, de lo exterior y lo interior. En este aspecto, dice

Correlativamente la conciencia para la cual la Gestalt existe, no era la conciencia intelectual, sino la experiencia perceptiva. Habría que interrogar a la conciencia perceptiva para encontrar en ella una aclaración definitiva. Limitémonos aquí a indicar cómo el estatuto del objeto, las

\footnotetext{
${ }^{4}$ En "Titres et travaux...", explicita que el filósofo que se pone a reflexionar acerca de la percepción se aparta del cuerpo en el cual él habita, e incluso de las cosas a las cuales el cuerpo se encamina en el curso de la vida, en verdad, se realiza como sujeto contemplativo. Las cosas percibidas, sucesivamente, se retiran de nosotros, volviéndosenos indiferentes, las cuales se hacen determinadas posteriormente por medio de algunas normas, algún conjunto de caracteres que podamos identificar en ellas. Simultáneamente, el cuerpo propio no es más que otro integrante de esas cosas u objetos, alzado en un tiempo posterior al señalado a la dignidad de saber científico, sin embargo de modo similar a ellos encaminado a una explicación por conexiones de función a variante. De cara a una conciencia de tipo filosófica en primera persona el sujeto trascendental -o el cual tiene la facultad de conocer-, el cual no es más que sujeto, se extiende un todo de objetos en tercera persona los cuales no son más que objetos (Merleau-Ponty, 2000).

${ }^{5}$ Más adelante explicitan: "[1]a percepción revela, es decir, permite que el ser se ilumine y dote con ello de sentido dado nuestro entorno. Se diría que el mundo se llena de sentido en virtud de una actividad perceptiva orquestada por nuestro cuerpo. Esta revelación de sentido no sería posible si la percepción no estuviera ligada al mundo como una totalidad, ya que somos como una continuación del mundo. Bajo esas condiciones, la percepción está encarnada en el corazón mismo del mundo, y, en el momento en que el hombre se prolonga a la realidad, realiza un quiasmo (cuerpo-X-mundo), pues lo que percibe es también percibido" (González y Jiménez, 2011, p. 118). Prosiguiendo en este contexto, Merleau-Ponty expone: “[1]a fenomenología, como revelación del mundo, reposa sobre ella misma, o más aún, se funda a sí misma" (1957, p. XIX).
} 
relaciones entre la forma y la materia, entre el alma y el cuerpo, la individualidad y la pluralidad de las condiciones, se fundan en ella. (Merleau-Ponty, 1953, p. 292)

De modo que lo fundamental se basa en vincular al sujeto y objeto por medio del cuerpo en un hecho de percepción, ya que el comportamiento humano en su conjunto se encuentra en cierto modo expuesto desde dentro. En la percepción, pues, el sujeto se dispone en un cuerpo, mostrándose inútil eludir la actuación del mundo como lugar de acto de la percepción, puesto que en el propio movimiento nos instala en su ámbito. ${ }^{6}$

\section{SOBRE LA IDEA DE ENCARNACIÓN}

La otra cuestión que nos interesa presentar es la idea fenomenológica de 'sujeto encarnado'. En una fenomenología del cuerpo se admite el ser encarnado como una distinción no sólo biológica del cuerpo del hombre. La postura del cuerpo como 'trascendental' se manifiesta más allá de la sola condición orgánica. En otras palabras, ambas cuestiones, tanto el cuerpo no entendido a modo de objeto como el ser trascienden al organismo, se revelan con la idea de encarnación. Por consiguiente, en este caso, la encarnación merleaupontyana trascendental tiene dos acepciones: una, como resultado de la encarnación, el cuerpo viviente sobrepasa sus límites de su significado objetivo hasta alcanzar ser la encarnación de un sujeto; otra, el espíritu como encarnado descubre el espíritu arraigado en un cuerpo. En definitiva, se trata de comprender de manera fenomenológica el cuerpo viviente. Cabe destacar la importancia de la noción encarnación en el compendio conceptual de Merleau-Ponty. Para nuestro filósofo la encarnación se encuentra íntimamente relacionada con la subjetividad, posicionando a ésta ante una nueva situación, en el cual al mundo se lo vive; y lleva en sí, además, la no admisión a la dualidad objetosujeto y a la noción de sujeto simple y único amarrada al 'yo pienso' cartesiano, cuyo sujeto se piensa disociado del mundo y del cuerpo (Gallo, 2006). "El mundo no es lo que pienso, sino lo que vivo, estoy abierto al mundo, comunico indiscutiblemente con él, pero no lo poseo, es inagotable" (Merleau-Ponty, 1957, p. XV). En realidad, el mundo no es un objeto del que tenga la norma de su constitución a través de la mediación de mi yo, el entorno natural y el ámbito de cada uno de mis pensamientos y de cada una de mis percepciones precisas. La verdad no 'habita' únicamente en el 'hombre interior', el hombre es en el mundo, y es en el mundo en el cual se conoce. "Este sujeto exterior sin interior, este yo que está siempre ya en el mundo o que 'es-del- mundo' es un sujeto esencialmente corporal" (García, 2012, p. 77). En la ocasión que vuelvo en mí desde un dogmatismo científico o del dogmatismo del sentido común, hallo no un centro de verdad interior, sino un sujeto dedicado al mundo. Merleau-Ponty, en fin, trata de encontrar en la idea de encarnación una 'unidad primaria'.

${ }^{6}$ Con respecto a esto, González y Jiménez agregan: “[e]l cuerpo humano es, visto desde los ojos de MerleauPonty, un 'donador de sentido"” (2011, p. 118).

${ }^{7}$ En referencia a esto, Gallo expresa: "Merleau-Ponty rechaza la relación meramente instrumental entre cuerpo y alma, y en sentido fenomenológico con la idea de sujeto encarnado, como espiritualización de la carneencarnación del espíritu, intenta dar cuenta de la imbricación originaria del cuerpo y del espíritu, que transforma 
De este modo queda en evidencia aquello que nosotros pretendemos mostrar, comprendiendo al ser humano como idea de sujeto encarnado, Merleau-Ponty no continúa con la corriente dualista del vínculo alma y cuerpo, porque desaparece las nociones de principio espiritual (alma) y material (cuerpo), enlazándose estos en la noción de encarnación.

Ahora bien, explicitado ambos puntos, retomemos el hilo conductor de nuestro trabajo. Conforme a que nuestros saberes del individuo toman otras connotaciones, creemos sospechar un vínculo diferente entre el individuo que es capaz de percibir y su cuerpo, además de estar en presencia de una nueva revelación del mundo que se percibe, conduciendo así a convocar a un análisis de nuestra idea de espíritu y de sujeto. En Fenomenología de la percepción, Merleau-Ponty dedica el tercer capítulo a "El cuerpo". Continuamos así nuestro desarrollo con la temática del cuerpo merleaupontyana.

\section{EL CUERPO COMO VEHÍCULO DEL SER EN EL MUNDO}

En Fenomenología de la percepción, Merleau-Ponty comienza a tematizar la idea de cuerpo del siguiente modo: "[n]uestra percepción termina en los objetos, y el objeto, una vez constituido, se presenta como la razón de todas las experiencias que de él hemos tenido o que podremos tener" (Merleau-Ponty, 1957, p. 73). Así, el filósofo pretende reflejar un modo específico de alcanzar al objeto, pues tanto mi propio pensamiento como mi modo de ver son ambas conocidas por mí claramente. Tenemos que comprender de qué manera el modo de ver se realiza a partir de algún lugar sin encontrarse contenido en su modo de dirigir la mirada. Este modo de dirigir la mirada no altera el modo con que busco ver el objeto pretendido, debido a que los objetos se descubren y velan a través de la misma vía. Ver, pues, es ingresar en un orbe de seres que muestran, los cuales no se exponen si no pueden resguardarse tras de mí o unos de otros. Es decir, ver un objeto es habitarlo y a partir de ahí aprehender todas de las cosas según la cara que al 'mismo' regresan. Cada objeto en su totalidad es el que da la imagen de todos los objetos restantes. Entonces, en este primer momento del análisis, Merleau-Ponty determina tratar al cuerpo como objeto, es decir, considera su cuerpo, el cual es su propia perspectiva acerca del mundo, como uno de los demás objetos de este mundo.

Cuando hablamos de cuerpo en sentido 'propio' se pretende decir que es un conglomerado de condiciones específicas bajo las cuales una idea existencial se concreta en algo de mi propiedad. Es destacable como Merleau-Ponty establece una semejanza entre el corazón y el cuerpo, puesto que el corazón está en el organismo como el cuerpo propio en el mundo, estimulándolo y sosteniendo con vigor la representación palmaria (MerleauPonty, 1957, p. 223). Siendo así, ¿qué nos quiere decir el filósofo francés con esto? Que el cuerpo es el vehículo del ser en el mundo. El cuerpo propio es, en consecuencia, el cuerpo ya invadido por las ideas existenciales. Merleau-Ponty, sobre eso, expresa que no puede

al uno de cuerpo materia en cuerpo sujeto y al otro de espíritu retirado en sí mismo, desencarnado, en espíritu encarnado" (2006, p. 49). 
comprender el cometido del cuerpo vivo excepto que lo realice uno mismo, como tampoco el modo de un cuerpo direccionado al mundo. Para ello, presenta un ejemplo sobre la amputación de una parte del cuerpo humano y el modo en que éste encierra la ambigüedad de la noción de comportamiento, donde los estímulos dan señales de esa parte faltante al sujeto, creyendo éste por tal caso una pierna fantasma. El ejemplo describe, en breve, cómo la estimulación suplanta a la pierna en la transmisión de información desde el muñón al cerebro y la que la recibe es el alma, ya que se encuentra en lo inmediato unida al cerebro y solamente a este. Merleau-Ponty encuentra en los fenómenos psicopatológicos un procedimiento que le sirve para aclarar ciertas cuestiones que tienen que ver con la conexión entre el cuerpo y el mundo. En el examen acerca del miembro fantasma y la falta de admisión del paciente ante la pérdida de una extremidad (anosognosia) tiene por objeto mostrar que indistintamente de los ejemplos seleccionados, para explicar válidamente estos, no sirven la fisiología mecanicista ni la psicología clásica. No obstante, la exposición del ser en el mundo nos orienta a entender algunos matices de estos ejemplos particulares que nos quedarían alejados de nuestra comprensión si fuera de otra manera (Gordillo, 2000, pp. 83-85). Así, pues, Merleau-Ponty expresa que para desarrollar la convicción tanto de la anosognosia como del miembro fantasma, Schilder, Menninger-Lerchenthal y Lhermitte toman a ambos fenómenos, encuadrados dentro de una noción de pensamiento orgánico, como una 'plegadura orgánica', desde aquí su posible vinculación de lo fisiológico y de lo psíquico. Por ejemplo, en el momento que el insecto reemplaza por medio de un acto irreflexivo la pata sana por la pata amputada, no pretende significar que un mecanismo de ayuda predeterminado reemplace por una actividad instantánea el miembro mutilado, como tampoco es cierto que el mismo animal sea consciente de llevar a cabo tal actividad, porque en el caso de tener la pata atada no reemplazaría la misma.

El animal sigue estando en el mismo mundo y se lanza hacia él con todas sus facultades. El miembro atado no es suplido por el miembro libre, porque continúa contando en el ser del animal, y la corriente de actividad que va hacia el mundo pasa todavía por él. (Merleau-Ponty, 1957, p. 84)

De este modo, Merleau-Ponty establece que la distinción se basa en que el animal orienta por sí las regulaciones de su ambiente y él mismo coloca los límites de su cuestión fundamental. Sin embargo no obedece a una opción particular, sino a un 'a priori' de la especie. De igual manera, el filósofo francés explicita que el movimiento del ser en el mundo es aquello que existe tras el fenómeno de reemplazo.

"Cuando se dice que un animal existe, que tiene un mundo, o que es en el mundo, no se quiere decir que tenga percepción o conciencia objetiva de él" (Merleau-Ponty, 1957, p. 84). La situación, que ocasionan las actuaciones irreflexivas, no se encuentra en su totalidad especificada y conectada, por consiguiente, ellas no brindan un sentido práctico, sino que convocan a un reconocimiento corporal. Efectivamente, debido a su propio desconocimiento, posibilita a los miembros reemplazarse uno por otro. Llama los movimientos del animal de un modo que se adaptan a una significación de la situación, por tal motivo, los reflejos jamás son transformaciones ciegas. Así, esa presencia general de la situación brinda un sentido a los estímulos fragmentarios y existencia para el organismo. El 
reflejo hace a los estímulos objetivos ser tal 'situación', se encuentra con ellos en un vínculo de conocimientos, entendiéndose así para su posterior confrontación.

Precisamente, aquello que permite realizar el entrelazamiento de lo 'fisiológico' y de lo 'psíquico', se debe a que 'el ser en el mundo' es capaz de diferenciarse de todo saber en primera persona y de todo desarrollo en tercera persona, efectivamente por ser un punto de vista 'preobjetivo'.

El reflejo, en tanto que se abre al sentido de una situación, y la percepción en tanto que no pone primeramente un objeto de conocimiento y en tanto que es una intención de nuestro ser total, son modalidades de una perspectiva preobjetiva, que es justamente lo que llamamos ser en el mundo. (Merleau-Ponty, 1957, p. 86)

Con todo, Merleau-Ponty prosigue el desarrollo de la idea sobre la no admisión de una explicación fisiológica o psicológica o mixta, aunque se pueda resumir a algunas de las dos condiciones. De hecho amplia con lo siguiente, la anosognosia, desde una explicación fisiológica, es la ausencia de una parte de la figuración del cuerpo que debería estar dado, ya que la extremidad correspondiente se encuentra ahí; por su parte, la extremidad fantasma es la presencia de un fragmento de la figuración del cuerpo que no debería darse, ya que la extremidad correspondiente no se encuentra ahí. Desde una explicación psicológica de ambos fenómenos, de la anosognosia se hace un olvido, algo imperceptible, siendo una ausencia efectiva de representación; de la extremidad fantasma un recuerdo, una percepción, siendo una presencia efectiva de representación. En ambas cuestiones, no nos podemos quitar del mundo objetivo. La extremidad fantasma se da en el orden de la presencia ambivalente de la extremidad, y no así en la representación de ella. En otras palabras, rechazar la deficiencia en la anosognosia o la mutilación de la extremidad no recae en una decisión deliberativa. Estos defectos se manifiestan por lo general de un modo rápido y se apropian del sujeto que se encuentra ya vuelto por el cuerpo a un mundo verdadero particular, además de ser direccionado a algún tipo de conducta. No obstante, este fenómeno se hace más comprensible desde la perspectiva del ser en el mundo. Aquello que en nosotros no acepta la deficiencia y la mutilación es un 'yo' implicado en concreto mundo interhumano y físico, que continúa así desplegándose a su mundo en contra de las mutilaciones o las deficiencias y, que de tal modo, no las admite de ley. Así, poseer un brazo fantasma representa estar abierto a cada una de las acciones del cual solamente el brazo es apto, pues, representa cuidar el campo objetivo que se tenía antes de la amputación. A partir de este punto de vista, hay que comprender la no admisión como una negación de nuestro mundo particular y un desplazamiento al mundo natural despojado de todo carácter personal.

Todo ser humano, en el mundo de su propia experiencia, por ser inherente a su cuerpo, apunta a un sitio de acciones contingentes que establecen su adecuación al mundo. Así, como ya hemos mencionado, el cuerpo es el medio del ser en el mundo, y para el ser viviente poseer un cuerpo significa juntarse a un medio establecido, mezclarse con ciertos propósitos, asumir un compromiso continuo con ellos. Por esto, la deficiencia nos quita del mundo propio en donde el sujeto ya está y al que se inclina incesantemente, cuya razón se debe a que es el mundo propio para el cual el cuerpo humano ha sido preparado. De esta 
manera, cuando mi mundo habitual estimula en mí voluntades habituales, coincidentemente, en el caso de estar mutilado, ya no puedo hacerlo. Así, pues, en mi cuerpo propio se establecen 'regiones de silencio'. El paciente sabe, precisamente, tanto de su falta como de su rechazo. Merleau-Ponty, a este respecto, dice

En el caso que nos ocupa, la ambigüedad del saber se reduce a esto, que nuestro cuerpo entraña algo así como dos capas distintas, la del cuerpo habitual y la del cuerpo actual. En la primera figuran gestos de manejo que han desaparecido de la segunda, y la cuestión de saber cómo puedo sentirme provisto de un miembro que no tengo ya de hecho se reduce a saber cómo puede el cuerpo habitual figurar como garante del cuerpo actual (Merleau-Ponty, 1957, p. 89).

Entonces, qué hacer ante la percepción de objetos manipulables, ya que no puedo manipularlos, pues, es necesario que renuncie a aquello manipulable para transformarse 'en lo que se puede' manipular. Cabe destacar que en este momento del desarrollo se quiebra el vínculo entre el mundo y el cuerpo, incluso ante la disputa del sujeto por evitarlo. Así pues, la conexión entre el mundo y el cuerpo se detiene por un cierto tiempo y de algún modo también la conexión de intenciones corrientes del sujeto con su propio mundo. Por lo que sigue, se necesita que el cuerpo propio sea percibido tanto como una experiencia simple e instantánea como vulgar y universal. La desestimación de la deficiencia es una amenaza a mi integración, a mi mundo particular. ${ }^{8}$ No es de extrañarse que el sujeto sufriente de un miembro fantasma se manifieste por medio de emociones, el cual el mismo continúa descubierto a aquellos actos para los cuales el miembro amputado era funcional. Este tipo de emociones ligadas a creer que el enfermo cuenta aún con el miembro amputado es lo que le impulsa a tratar sostener el cuerpo funcional que poseía antes de la amputación.

Mientras que habito un mundo tangible en el cual se proporcionan situaciones corrientes y estímulos incesantes, nuestra propia vida conlleva órdenes que encuentran su naturaleza en el ambiente vulgar que nos cerca. Así, se manifiesta alrededor de nuestro existir individual una frontera poco más o menos vulgar. Al ser la existencia individual esporádica, hace que la decisión solamente puede otorgar a nuestro modo de vivir un sentido forzado. La anexión del cuerpo y el alma en la acción, de una vida biológica en una presencia individual, del orbe natural en orbe cultural, se concreta simultáneamente frágil y posible por la configuración temporal de nuestra experiencia. A Merleau-Ponty le basta este tratamiento para mostrar, por el momento, que pueden pensarse las conexiones entre lo fisiológico y lo psíquico. Para ello, apela a indagar la razón de por qué las rememoraciones que se provocan en el cercenado son capaces de manifestar el miembro fantasma. El miembro fantasma no es un recuerdo, es por poco presente. El cercenado lo percibe ahora, por ejemplo, el brazo replegado sobre su tórax, sin ningún registro de pasado. "El brazo fantasma es, pues, algo así como la experiencia reprimida de un antiguo presente que no se decide a convertirse en pasado" (Merleau-Ponty, 1957, p. 93).

\footnotetext{
${ }^{8}$ En este sentido, Gordillo, amplia: "[1]a actitud del sujeto consiste en tratar de eliminar las trabas que le impiden acceder a su mundo personal y le dirigen hacia el mundo impersonal del Umwelt, que está siempre ahí como sustrato" (2000, p. 85).
} 
En el caso de que la emoción sustituya al ser en el mundo, se entiende, explicita, que consiga estar en el comienzo del miembro fantasma. Si tanto la emoción como el recuerdo son capaces de manifestar el miembro fantasma, esto se debe a que por medio de una conducta existencial estimula a la otra, es decir, el recuerdo, la emoción y el miembro fantasma son semejantes condicionalmente al ser en el mundo. Por consiguiente, el ser humano puede obtener el espacio práctico e intelectual, desistiendo a una parte de su naturalidad e implicándose en el mundo a través de circuitos predeterminados y órganos estables, que en un comienzo lo irá a desligar de su ambiente y se lo hará mirar. Es más, Merleau-Ponty agrega que es inclusive una necesidad intrínseca de la existencia más incorporada darse un cuerpo habitual. Aquello que nos deja juntar lo 'psíquico' con lo 'fisiológico' expone que, reincorporados al existir, ambos se encuentran enfocados a un mundo o a un polo intencional, el cual ambos ya no se diferencian como el orden del para sí y el orden del en sí. Para Merleau-Ponty, de este modo, hay una sola explicación válida a estos ejemplos, pues, consiste en presentar las distintas maneras de ser en el mundo, afirmando que solamente la conciencia encarnada es la que puede verdaderamente explicitar este tipo de fenómenos.

El ser humano, en concreto, no consiste en cuestiones relativas a la mente entremezcladas a un organismo, es más bien este movimiento de la existencia que, en ciertos momentos, cede a que la corporicen y, en otros, se dirige a hechos individuales. MerleauPonty indica que las causas psicológicas y las ocasiones corporales pueden mezclarse, debido a que no existe un único movimiento en un cuerpo viviente "... que sea un azar absoluto con relación a las intenciones psíquicas, ni un solo acto psíquico que no encuentre cuando menos su germen o su trazado general en las disposiciones físiológicas" (MerleauPonty, 1957, p. 95). Así, Merleau-Ponty basa su fundamento acerca de concebir un punto de fusión entre lo psíquico y lo físiológico en una cita de Eric Menninger-Lerchental (Merleau-Ponty, 1957, p. 96, n. 24), pues, afirma que no se puede seguir pensando lo físico y lo psíquico por separado, es decir, en consumar uno de los dos campos prácticos con el otro, porque ambos están estrechamente unidos, y de este modo, ambos tienen que ser readmitidos por un tercero. Esta exposición le basta al fenomenólogo francés para demostrar que, a través de la físiología, la existencia se da en el cuerpo.

Efectivamente, el análisis de los comportamientos perceptivos llevados a cabo en la Fenomenología de la percepción permitió situar y enfocar el examen de la particular conexión del sujeto-cuerpo-mundo. Es, pues, por medio de la situación del cuerpo propio que somos capaces de captar el espacio exterior. A cada sucesión de ahora aquello que nos otorga un esquema corporal es una idea universal objetiva y tácita de los vínculos del cuerpo propio y de las cosas. Gordillo, en este sentido, expone

El esquema corporal es, entonces, la vivencia de nuestro cuerpo, a modo de estructura única sensomotriz. Es la vivencia que un sujeto tiene de sus posturas, movimientos actuales y posibles. Esta toma de conciencia global de las distintas partes de mi cuerpo y de sus diversas posturas se corresponde con los "proyectos" que tiene el organismo. Por ello, la vivencia de cada parte, ligada con las de todas las demás partes del cuerpo, hace posible conocer en cada momento los movimientos actuales y posibles de nuestro cuerpo. (Gordillo, 2000, p. 83) 
García, sigue en esta línea, y agrega

El cuerpo es vivido como la posibilidad de realizar tales y cuales movimientos o comportamientos en el mundo. El cuerpo tal como de él tengo experiencia instantánea no se corresponde exactamente con las características que un tercero puede observar de mi cuerpo ni con la imagen que yo podría observar de mi cuerpo en un espejo. Tampoco tengo experiencia del cuerpo anátomo-fisiológico tal como sería descripto por el científico: del funcionamiento total del sistema circulatorio, de mis órganos internos, de las sinapsis neuronales, etc. Tampoco es mi experiencia corporal en primera instancia una mera imagen psíquica o mental de mi cuerpo que contemplo con el "ojo de la mente". En cambio siento mi cuerpo instantáneamente como el habitante activo de un entorno pragmático; me vivo a mí mismo corporalmente como estando en tal posición, sentado aquí y con tales comportamientos posibles ofrecidos a mis manos, mis pies o mis ojos, lo cual es decir: como "esquema corporal" o "motriz". (García, 2012, p. 107)

Merleau-Ponty, de igual modo, presenta a la motricidad como una función sólidamente nerviosa y perceptiva. Más adelante, en "Titres et travaux...”, desplegará la idea que todo movimiento del cuerpo propio tiene lugar sobre el suelo de un cierto "proyecto motor'. Y en el momento que, cuando por ejemplo, ese proyecto motor cambia, de un movimiento de tomar (Greifen) a un movimiento de designar (Zeigen), los postulados del proceso motor son distintos, incluso si se tratasen de los mismos músculos afectados (Merleau-Ponty, 2000). Así, pues, movimiento y proyecto motor conforman un solo fenómeno, donde mis movimientos son modos diversos de la conexión universal en el mundo de la que el vehículo es mi cuerpo, y por tanto, no son para mí meros desplazamientos prácticos a los que concurro.

Al señalar que esparcimos al medio un 'tejido' de proyectos motores, MerleauPonty pretende afirmar que nuestro cuerpo habita el espacio. Nuestro cuerpo se adapta, en efecto, tal como la mano al útil. Y esto obedece a que al momento de pretender movernos de un punto a otro, desplazamos a nuestro cuerpo sin útiles, por ser este nuestro, y en virtud de éste obtenemos un acceso inmediato al espacio. En una publicación posterior, MerleauPonty expresará que el cuerpo tiene para nosotros una mayor significación que un medio o un útil, es la representación visible de nuestras intenciones, es nuestra expresión en el mundo. Queda claro, pues, que nosotros no nos trasladamos igual que una cosa. De hecho, conformamos o bosquejamos nuestra percepción de las cosas por medio de la ayuda que nos dan los "movimientos emotivos íntimos", movimientos intrínsecamente configurados por estados de ánimos (Merleau-Ponty, 2000, pp.36-48). Supuesto esto, para nuestros fines, consideramos importante analizar y explicitar la revisión conceptual que realiza Bernhard Waldenfels del concepto 'cuerpo', puesto que considera la no posibilidad de pensar el habitar en el espacio sin una pertenencia íntima de los habitantes al lugar en el cual están situados.

6. HABITAR, CORPORALIDAD, ESPACIO

En "Habitar corporalmente en el espacio", Waldenfels, dice: “[n]o se puede pensar el habitar en el espacio sin una pertenencia intrínseca de los habitantes al lugar en que se 
encuentran" (Waldenfels, 2015, p. 182). Efectivamente, Waldenfels afirma que quien carece de toda experiencia particular de saber el lugar dónde se halla el que dice 'aquí', consecuentemente, no capta aquello que la misma expresión pretende significar. En verdad, un 'aquí' -dice- carente de situación quedaría a modo de un gesto lingüístico vacuo, donde se puede usar el ejemplo que el usuario de un mapa de ruta no sabría su lugar de emplazamiento de acuerdo al contenido enunciativo vacío, consiguientemente, no tendría un sitio de referencia deíctico. El 'aquí' señaliza un instante del enunciado (énoncé) en que la enunciación (énonciation) presenta el lugar donde uno se encuentra simbolizado como un punto rojo en el mapa de ruta. La determinación del quién como del dónde de un hablante que se expresa aquí y en cualquier lugar no pueden escindirse. Así, podemos establecer un primer vínculo entre corporalidad y espacialidad, en el caso que nuestra reflexión encuentre como 'expresión de una corporalidad' que no sea propia de una sola cosa ni de un simple espíritu. Inclusive podemos ver cómo este vínculo halla en el 'aquí' su lenguaje, su expresión lingüística.

Tomado como eje espacial, el 'aquí corpóreo' actúa de igual modo como eje de situación de inicio de distintos ejes, por ejemplo: izquierda-derecha, arriba-abajo, delanteatrás. Así, en clara referencia al eje de coordenadas matemático, el 'aquí espacial' husserliano estructura un 'punto cero', o sea, el 'aquí' no señala ningún punto. Precisamente, 'punto cero' significa que señala el sitio de nacimiento de una estructura espacial ligado a sus significados e implicaciones, por tal razón, no quiere señalar ningún lugar en el espacio. Asimismo, este "punto cero" se equipara al punto cegado de la vista, en el cual el 'ver' tiene lugar, incluso el 'hablar' lo tiene. Por tal motivo, éste sitio se apropia los atributos de un nolugar y alude, así, a la extrañeza (fremd $)^{9}$ del lugar. En ese marco, dice: “[1] os ejes espaciales conforme a los cuales se orienta la situación espacial no forman, en efecto, una estructura espacial rígida a la que nuestro cuerpo estuviera sujeto como a una rejilla" (Waldenfels, 2015, p. 186). Las direcciones se generan por medio de un movimiento de 'orientarse a', en la cual una dirección espacial puede transformarse en otra. En consecuencia, el orientarse se encuentra sometido a una transformación abierta que por medio de derivaciones es expulsado continuamente fuera de curso.

El 'aquí' deja de ser una vaga relatividad en el momento que alguien o algo está en su sitio. Así, pues, el 'aqui' se transforma específicamente en lugar de estancia. El hablante encuentra el lugar de referencia en el cual dispone de lugar el discurso del 'aquí'. Waldenfels establece, en tal sentido, que la distinción que no se puede acotar en la relación dentro-fuera está ya supuesta en la especificación de la espacialidad como exterioridad. Lingüísticamente, el dentro se encuentra señalado. El dentro es el lugar donde algo o alguien 'se' aleja. "La delimitación hacia dentro comienza, una vez mas, en el propio cuerpo, con la piel como superficie límite y de contacto. La esfera corporal interna se extiende" (2015, p.

\footnotetext{
${ }^{9}$ Por una cuestión de enfoque y de cierta delimitación en nuestro trabajo, no ahondaremos en el concepto de 'extrañeza', mencionado por Waldenfels. Creemos que la conceptualización merece un tratamiento diferenciado, por tanto, excede al presente trabajo. A modo de referencia, referirse al capítulo "La extrañeza del cuerpo propio" (véase Waldenfels, 2015, pp. 53-72).
} 
188). Asimismo es común referirse a los artefactos como expansión del cuerpo, la morada supone una prolongación del ámbito corporal propio e íntimo. Por ejemplo, el muro y la pared, como elementos divisorios, suponen implícitamente la distinción dentro-fuera, siendo éste y otros límites problemas que solamente se despliegan en la superficie de una cierta 'place identity' ('identidad del lugar'). Cabe aclarar que el término 'place identity' se refiere en este contexto a los límites a determinar y a su propia movilidad, permeabilidad, trazabilidad y permanencia.

Siempre que el 'aquí corpóreo' nos signifique tanto el lugar en que se encuentra fijada cualquiera de las 'orientaciones a', como el lugar a partir del cual se inicia cada uno de nuestros movimientos en el espacio, hay que agregar que en tanto existencias corporales, fijamos nuestra permanencia en una posición que retrocede a un movimiento que se ha paralizado. De este modo, esta abertura interior del 'estar aquí' nos lleva a que la propia existencia espacial se desplace de una punta a la otra. La concerniente situación de cada movimiento queda inserta en una doble oscilación de los extremos, ya que puede quedar disipado en una espacialidad borrosa o estrecharse en un lugar anclado, pues, la doble oscilación dirige a una debilidad concentrada del 'aquí corpóreo' que se acerca casi al traspasar el extremo. "En este ir y venir extremos se manifiesta la duplicidad de nuestro cuerpo, que como cuerpo (Leib) nos abre y proporciona un mundo en el que él aparece al mismo tiempo como un cuerpo (Körper)" (Waldenfels, 2015, pp. 191-192). ${ }^{10}$ La modalidad de ser del cuerpo radica justamente en estar simultáneamente 'aquí' y desplazado de éste 'aquí'. Así, por haber adjudicado al sí mismo espacial una 'place identity', de igual manera, hay que asignarle una 'place non-identity'. Efectivamente, la localidad que siempre habitamos como lugar se combina con una alocalidad, pues, justamente, esto establece la posibilidad de distintas heterolocalidades que no permiten ingresar por medio de la violencia en una unitaria configuración de coordenadas, ni tampoco se permite mezclar unas con otras.

Asimismo, si lo reflexionamos desde de la perspectiva temporal, el lugar en el cual ya estuve adquiere cuerpo en un 'aquí', en tanto que el lugar donde puedo estar refiere a un espacio lúdico de posibilidades venideras. Pues, la existencia espacial adquiere con esto una densidad temporal. Durante la historia de un cuerpo, “... el aquí actual del cuerpo actual se densifica en el aquí habitual de un cuerpo habitual. La orientación corporal y los movimientos corporales están anclados en la habituación del cuerpo" (Waldenfels, 2015, p. 192). Queda asentado esto como cierto en las ocasiones en las que nos dirigimos de manera voluntaria.

Ahora bien, por ejemplo, en los casos clínicos es adonde encontramos este doble aumento a los límites: por un lado, el enfermo tiene saberes, por ejemplo, conoce las letras del abecedario, aunque hay casos que no saben de qué modo aplicarlas; por otro lado, para ubicarse en el espacio, el enfermo no puede deshacerse de las especificaciones puntuales

\footnotetext{
${ }^{10}$ Leib es el vínculo físico del sujeto con la realidad pero el Körper es aquello que se puede captar y medir objetivamente mientras que el Leib no puede tener esa objetivación fácilmente. Con el fin de profundizar la distinción de cuerpo como Leib o Körper, remitirse la nota del traductor. Véase Waldenfels (2015, p. 177, n. 1).
} 
que muchas veces obtiene por medio de la manipulación, entonces para realizar algún tipo de indicación, continúa con el acompañamiento una representación visual con la mano. En estos casos, el conocimiento intelectual y la actividad motriz van desajustados uno de lo otro. Precisamente, concatenando con los casos clínicos, Merleau-Ponty explicita que el paciente sabe dónde se encuentra, por ejemplo, identifica su pipa por un 'saber absoluto', por lo tanto, sabe en qué lugar se encuentra su cuerpo y su mano. Expresa

La palabra 'aquí', aplicada a mi cuerpo, no designa una posición determinada en relación con otras posiciones o en relación con coordenadas exteriores, sino la instalación de las primeras coordenadas, el anclaje del cuerpo activo en un objeto, la situación del cuerpo frente a sus tareas. (Merleau-Ponty, 1957, p. 107)

Así pues, tras considerar el cuerpo como movimiento, Merleau-Ponty expresa que el movimiento recupera al espacio y tiempo en su significación primaria, que resulta disimulada en la futilidad de las situaciones obtenidas. De hecho, la motricidad no actúa de asistente de la conciencia trasladando el cuerpo a la referencia del espacio que en un primer momento nos habíamos figurado. Es necesario que primeramente el objeto exista para el cuerpo, para que se pueda así mover su cuerpo en dirección a un objeto. En este sentido, Merleau-Ponty afirma: "[n]o hay, pues, que decir que nuestro cuerpo está dentro del espacio, ni, por otro lado, que está dentro del tiempo: habita el espacio y el tiempo" (Merleau-Ponty, 1957, p. 152).

Mientras que poseo un cuerpo y actúo por medio del cuerpo en el mundo, implica que soy en el espacio y tiempo, además de comprenderlos, mi cuerpo se adapta al espacio y tiempo. La capacidad de este comprender calcula la de mi existencia, sin embargo, no puede ser jamás absoluta. El tiempo y el espacio que yo habito poseen continuamente confines indefinidos que comprenden otras perspectivas. La síntesis tanto del espacio como del tiempo tiene que ser siempre reiniciada.

La experiencia motriz de nuestro cuerpo no es un caso particular de conocimiento; más bien nos procura una manera de acercarnos al mundo y al objeto, una "praktognosia" que ha de ser reconocida como original, y, quizá, como originaria. Mi cuerpo tiene su mundo, o comprende su mundo, sin tener que pasar por "representaciones", sin subordinarme a una "función simbólica" u "objetivante" (Merleau-Ponty, 1957, p. 153).

Merleau-Ponty explicita que los psicólogos en reiteradas veces expresan que el esquema corporal es 'dinámico'. Por 'dinámico' entiende que mi cuerpo se me manifiesta a modo de postura en vista de alguna tarea posible. Precisamente, su espacialidad no se condice con una espacialidad de posición, como la de los objetos exteriores, sino con una espacialidad de situación. Por tal motivo, el esquema corporal es un modo de expresar que mi cuerpo es en el mundo. El esquema corporal es, precisamente, un sistema de correspondencias, por medio de la cual los distintos quehaceres cinéticos son desplazados ipso facto. En otras palabras, lo que les otorga un significado motriz a las consignas verbales es que es una experiencia de mi cuerpo y, a su vez, una experiencia de mi cuerpo en el mundo. Por lo tanto, la función cinética es la función deshecha en los desórdenes apráxicos. 


\section{A MODO DE CIERRE}

En el presente trabajo tratamos de explicitar el modo fenomenológico de concebir el sujeto de la encarnación, a partir de un análisis del cuerpo, y la relación el cuerpo y el mundo. Queda en evidencia que Merleau-Ponty considera que la conexión entre el cuerpo y el mundo es una disposición existencial que determina la significación de los actos psicológicos y no una conexión que remite a una causa. Todo el proceso está conformado por distintos niveles de la existencia, que muestra la existencia de una totalidad que se integra a medida que se lleva a cabo el proceso.

Precisamente, por ello, se puede comprender al cuerpo como el requisito de la conciencia, puesto que el soma del individuo se encuentra ya como sujeto dialéctico con el mundo y el resto de las cosas. De modo que, el cuerpo es la modalidad en que llegamos al mundo, del mismo modo que es el lugar de manifestación del mundo que estaba oculto a nuestra vista. Esto se debe a que tanto la conciencia como el mundo pasan por medio del cuerpo. Así, pues, este análisis corresponde a un doble examen, el del mundo que percibe y el del mundo percibido. En efecto, Merleau-Ponty pretende señalar que hay una correlación afianzada en la conexión de mi cuerpo y el mundo, entre mis actos corpóreos y el mundo. Merleau-Ponty piensa, de igual manera, que el cuerpo puede ser verdaderamente el vehículo del 'ser en el mundo', es decir, la condición de la existencia y del lugar. Establece, por consiguiente, que a través del cuerpo yo me sitúo en el mundo y al descubrirme permanezco abierto al mundo mismo que es ineludible para realizar mis posibles actos corpóreos. Al ser el cuerpo el lugar de vivos significados es de esperar que, asimismo, el cuerpo sea la posibilidad de comprender las cosas y de tener el mundo.

De este modo, con la noción fenomenológica de sujeto encarnado, es manifiesto que el filósofo francés se instala en el límite de un lenguaje involucrado en una búsqueda de otro tipo de ontología (quizás más próxima a una 'nueva ontología'), abierta por la misma percepción. En consecuencia, podemos pensar a partir del enfoque filosófico propuesto por Merleau-Ponty, otro modo de situarnos en la reflexión filosófica y reflexionar así la conexión mundo-cuerpo-cosas-y-seres, mediante la percepción, bajo otra perspectiva. De hecho, Merleau-Ponty a la temporalidad de la existencia la instala en la corporalidad, estableciendo así una nueva problemática dentro de los estudios fílosóficos contemporáneos: el sujeto encarnado como unidad de análisis filosófico.

\section{SOBRE EL AUTOR}

En la actualidad es licenciado de Filosofía por la Universidad Nacional de San Martín. El interés filosófico se centra en torno al pensamiento de Martin Heidegger y cuestiones metafísicas. Ha sido adscrito a la asignatura Seminario, a cargo del Dr. A. Bertorello (UNSAM); actualmente ayudante ad honorem de la materia Psicología, Ética y Derechos Humanos (UBA). Es miembro adherente de la Sociedad Iberoamericana de Estudios Heideggerianos (SIEH). Colabora, además, del Seminario de investigadores en formación, 
coordinado por el Lic. Ariel Vecchio, espacio dependiente del Centro de Estudios de Hermenéutica (UNSAM). Ha participado como asistente y ponente, organizador y colaborador en congresos internacionales y jornadas nacionales. Ha participado como organizador del ciclo "Charlas UNSAM". Tiene publicaciones nacionales. Forma parte del proyecto de reconocimiento interno (PRI) 2021-2022 (bienal): El Ereignis como espacio de juego, a cargo del Dr. A. Bertorello (titular) y del Lic. Ariel Vecchio (Cotitular), UNSAM.

\section{BIBLIOGRAFÍA}

Gallo, L. (2006). El ser-corporal-en-el-mundo como punto de partida en la fenomenología de la existencia corpórea. En Pensamiento Educativo, vol. 38, pp. 46-61.

García, E. (2012). Maurice Merleau-Ponty. Filosofia, corporalidad y percepción. Buenos Aires: Rheis.

González, R. y Jiménez, G. (2011). Fenomenología del entrecruce del cuerpo y el mundo en Merleau-Ponty. Ideas y Valores, $N^{\circ}$ 145, Bogotá, pp. 113-130.

Gordillo, L. (2000). El momento existencial del Cuerpo en Merleau-Ponty. Daímon, Revista de Filosofia, $N^{\circ} 20$, Murcia, pp. 75-88.

Merleau-Ponty, M. (1953). La estructura del comportamiento. Buenos Aires: Librería Hachette S.A.

Merleau-Ponty, M. (1957). Fenomenología de la Percepción. México: FCE.

Merleau-Ponty, M. (1969). Filosofia y lenguaje. Collège de France, 1952-1960. Buenos Aires: Editorial Proteo.

Merleau-Ponty, M. (2000). Parcours deux 1951-1961, Lonrai: Verdier.

Ralón de Walton, G. (2015). La "animalidad" como instancia originaria de la existencia Humana. En L. Basso y F. Giorgini (eds.), Actas de las XIV Jornadas Nacionales Agora Philosophica y III Coloquio de Hermenéutica: Merleau-Ponty en discusión (pp. 164-177). Mar del Plata: Universidad Nacional de Mar del Plata; Ciudad Autónoma de Buenos Aires: Asociación Argentina de Investigaciones Éticas, Regional Buenos Aires.

Waldenfels, B. (2015). Exploraciones fenomenológicas acerca de lo extraño. Barcelona: Anthropos Editorial; Morelia (México): Universidad Michoacana de San Nicolás de Hidalgo. 